\title{
Meta
}

Journal des traducteurs

Translators' Journal

\section{Visuelle Komik: Sprache, Bild und Typographie in der Übersetzung von Comics}

\section{Klaus Kaindl}

Volume 53, numéro 1, mars 2008

Le verbal, le visuel, le traducteur

The Verbal, the Visual, the Translator

URI : https://id.erudit.org/iderudit/017978ar

DOI : https://doi.org/10.7202/017978ar

Aller au sommaire du numéro

Éditeur(s)

Les Presses de l'Université de Montréal

ISSN

0026-0452 (imprimé)

1492-1421 (numérique)

Découvrir la revue

Citer cet article

Kaindl, K. (2008). Visuelle Komik: Sprache, Bild und Typographie in der

Übersetzung von Comics. Meta, 53(1), 120-138. https://doi.org/10.7202/017978ar
Résumé de l'article

Longtemps, la recherche sur la traduction de l'humour dans les bandes dessinées s'est concentrée sur la dimension linguistique en négligeant les dimensions pictorales et typographiques. Le présent article esquisse tout d'abord les différentes techniques humoristiques et ses fonctions pour le récit. Après avoir spécifié les relations entre l'humour et le comique, cet article propose une définition plus large de l'humour comprenant ses composantes linguistiques et ses élements non verbaux. La démarche adoptée étant nécessairement interdisciplinaire, l'auteur examine le phénomène de l'humour dans la bande dessinée de façon holistique et établit différentes catégories humoristiques qui sont illustrées par de nombreux exemples. 


\title{
Visuelle Komik: Sprache, Bild und Typographie in der Übersetzung von Comics
}

\author{
KLAUS KAINDL \\ Universität Wien, Wien, Austria \\ klaus.kaindl@univie.ac.at
}

\begin{abstract}
RÉSUMÉ
Longtemps, la recherche sur la traduction de l'humour dans les bandes dessinées s'est concentrée sur la dimension linguistique en négligeant les dimensions pictorales et typographiques. Le présent article esquisse tout d'abord les différentes techniques humoristiques et ses fonctions pour le récit. Après avoir spécifié les relations entre l'humour et le comique, cet article propose une définition plus large de l'humour comprenant ses composantes linguistiques et ses élements non verbaux. La démarche adoptée étant nécessairement interdisciplinaire, l'auteur examine le phénomène de l'humour dans la bande dessinée de façon holistique et établit différentes catégories humoristiques qui sont illustrées par de nombreux exemples.
\end{abstract}

\begin{abstract}
Humour is an important aspect of many comics. Nevertheless, the vast majority of contributions to the translation of comics make no attempt to categorize or to define humour and its function for the genre. A first step in discussing comics and translation should be to identify various sub-genres of comics and to specify the categories and interrelations of "humour" and "comic." In the few works on the translation of comics, the examination of the humorous dimension tend to be limited to verbal humour, i.e., playing with names and puns. The examples are almost always taken from "linguistically demanding" comics such as Astérix or Tintin. After a detailed review of the few aspects treated in linguistic, semiotic and literary articles on the translation of comics, the author evinces an approach which encompasses not only the verbal but also the non-verbal dimension of humour in comics. With the help of semiotic theories, the concept of humour is extended to the graphical and visual dimensions to provide a comprehensive account of humour in comics.
\end{abstract}

\section{MOTS-CLÉS/KEYWORDS}

visuelle Komik, Zeichenspiele, Comics, Typographie, Onomatopoien

Wissenschaft ist ein ernstes Geschäft. Die Ernsthaftigkeit der Wissenschaft scheint dabei der Auseinandersetzung mit dem Unernsten entgegenzustehen, dem in der Wissenschaft häufig der Geruch des Unseriösen anhaftet. Dies mag auch ein Grund sein, warum Comics verhältnismäßig selten Gegenstand wissenschaftlicher Arbeiten sind. Zum einen handelt es sich um eine hybride Gattung, die keiner wissenschaftlichen Disziplin eindeutig zugeordnet werden kann. Zum anderen werden Comics häufig - nicht zuletzt aufgrund der Gattungsbezeichnung - mit komischen und humorvollen Inhalten verbunden. 
Tatsächlich hatten Comics, in den ersten Jahren nach ihrer Erfindung 1895 in den USA primär komische Geschichten zum Gegenstand und inspirierten sich an politisch-sozialen Karikaturen, wie sie etwa in England seit dem 18. Jahrhundert bekannt waren, und an deutschen Bildergeschichten eines Wilhelm Busch. Sowohl der Alltag der „kleinen Leute“, der bis 1915 das zentrale Thema vieler Comic strips bildete als auch die ab 1915 beliebten Familiengeschichten wurden in der Regel auf humorvolle Weise dargestellt. Erst gegen Ende der 20er, Anfang der 30er Jahre entstanden vermehrt Comics, die nicht dazu gedacht waren, die Leser zum Lachen zu bringen, sondern auf Spannung, Abenteuer oder Gruseleffekte setzten. ${ }^{1}$ Die inhaltliche Nähe zu Komik und Humor, die der Gattung ursprünglich ihren Namen gab, konnte damit nicht länger als ein definitorisches Merkmal von Comics erachtet werden. Was die verschiedenen Subgenres (wie z.B. funny comics, Abenteuer-, Grusel, Superheldencomics etc.) vereint, sind ihr narrativer Charakter sowie die Darstellung der Handlung mittels sprachlicher, graphischer und/oder bildlicher Zeichen in einer Reihe von (mindestens zwei) gezeichneten Einzelbildern. Die verschiedenen Gestaltungsmittel, derer sich Comics bedienen und die von den verschiedenen sprachlichen Elementen, wie Sprechblasen, Erzähltexte, Onomatopoien, Bildinschriften über typographische Elemente wie Schriftart, -größe und (pikto)graphische Elemente, wie z.B. speedlines, und Ideogramme, wie Sterne, Blumen etc. bis hin zu den bildlichen Darstellungen von Personen, Gegenständen und Situationen reichen, ${ }^{2}$ sind alle an der Bedeutungskonstitution beteiligt. Die semiotische Komplexität wird in den einzelnen Subgenres dazu eingesetzt, um Spannung, Gruseleffekte oder eben auch Humor und Komik zu erzeugen.

Im folgenden soll untersucht werden, inwieweit Komik und Humor in übersetzungswissenschaftlichen Arbeiten über Comics berücksichtigt werden, welche Aspekte behandelt werden, welche Defizite die bestehenden Arbeiten aufweisen und welche Faktoren berücksichtigt werden müssen, um eine umfassende Beantwortung der Frage, wie Komik und Humor im Comic hergestellt werden, zu ermöglichen.

\section{Literaturüberblick: Komik und Humor in der Comicübersetzung}

Generell wird in den Arbeiten zur Comicübersetzung weder der Zusammenhang zwischen Humor und Komik problematisiert noch wird der Versuch unternommen, das spezifisch Komische oder Humorvolle definitorisch zu erfassen. Dies scheint mir jedoch notwendig, zumal in der humortheoretischen Literatur unterschiedliche Begriffshierarchien und Definitionen existieren. In einer Reihe von Ansätzen stellt Humor den Oberbegriff für verschiedenste Phänomene dar, wie etwa Witze, Scherze, Komödie, Parodie, Farce, Herumalbern etc. ${ }^{3}$ Andere wiederum erachten Komik als Oberbegriff für alle Bereich scherzhafter Kommunikation (z.B. Schmidt 1976). Die Feststellung, dass eine allgemeine Definition und Kategorisierung der verschiedenen Formen scherzhafter Kommunikation unmöglich ist (vgl. u.a. Attardo 1994: 3ff.; Schmidt, 1976: 168f.), ist dabei auf die Tatsache zurückzuführen, dass Komik und Humor entscheidend von den jeweiligen kulturellen Kontexten abhängen und somit immer nur in Abhängigkeit von den geschichtlichen und soziokulturellen Gegebenheiten fassbar sind. Das Verstehen von Humor und Komik ist somit nicht primär eine sprachliche Aktivität, die durch textimmanente Modelle erfasst werden kann, wie diese etwa Raskin (1985) versucht, sondern eine kulturelle Aktivität, die aus der 
kreativen Bisoziation verschiedener Wissensbereiche resultiert. Unter "bisociation" ist dabei ,the perceiving of a situation or idea (...) in two self-consistent but habitually incompatible frames of reference" zu verstehen (Koestler 1964: 35). Allgemein können Humor und Komik als Kommunikationsformen bezeichnet werden, die mittels komplexer Inferenzen funktionieren und ihre Wirkung primär daraus beziehen, dass sie nicht auf eine einzige Bedeutung festgelegt werden können, sondern gezielt mit simultanen Doppelassoziationen arbeiten, in denen der Rezipient den Bezugsrahmen einer Äußerung verdoppelt bzw. mit einem anderen Bezugsrahmen koppelt, wodurch es zu Doppeldeutigkeiten, Erwartungsbrechungen und plötzlichen Kontextwechseln kommt. Der wesentliche Unterschied zwischen Humor und Komik besteht laut Eco (1986) nun darin, dass letztere auf der Wahrnehmung von Gegensätzen, Humor hingegen auf der gefühlsmäßigen Erfassung dieser Gegensätze beruhe. Humor wäre demnach die Gefühlslage, die einen dazu befähigt, Komik zu erkennen. Im Gegensatz zum Witz, als zentralem Untersuchungsgegenstand der Humorforschung, bedarf Komik nicht der Pointe, sondern kann, wie Freud (1985: 171) feststellte, in der Wahrnehmung beliebiger Dinge entdeckt werden.

Obwohl in Comics durchaus auch Witze vorkommen können, so sind es doch vor allem Techniken des Komischen, die in dieser Gattung zum Einsatz kommen. Die Auseinandersetzung mit diesen Techniken und die Konsequenzen für die Übersetzung werden in der einschlägigen Literatur in unterschiedlichem Ausmaß wahrgenommen. Mounin kommt das Verdienst zu, die Comicsübersetzung als einer der ersten als relevanten Gegenstand wahrgenommen zu haben. Seiner Einladung an die Fachleute (1967: 135), Forschungen auf diesem Gebiet durchzuführen, wurde allerdings bis heute kaum Folge geleistet. Obwohl die Problematik der Übersetzung zwar häufig in literaturwissenschaftlichen, publizistischen, semiotischen und pädagogischen Arbeiten über Comics gestreift wird, existieren bis heute relativ wenige Arbeiten, in denen die Comicübersetzung den zentralen Gegenstand bildet. Die dabei behandelten Themen sind - je nach fachlicher Richtung, Fragestellung und Untersuchungsziel - breit gestreut und reichen von der Äquivalenzdiskussion (Würstle 1991) über die semiotische Komplexität von Comics und ihre Auswirkungen auf die Übersetzung (Schmitt 1997, Kaindl 1999a), die Rolle des Bildes für der Bedeutungskonstitution (Celotti 1997, Kaindl 1999b), die Übersetzungs- und Verlagspraxis (Schwarz 1989, Hunoltstein 1996) bis hin zur die pädagogischen Verwertbarkeit der Comicsprache (Lannoë 1991). Diese Ansätze werden im folgenden nur insoweit berücksichtigt, als sie auch die Übersetzung von Komik zum zentralen Gegenstand haben.

Jene Arbeiten, die sich mit Aspekten der Komik in der Comicübersetzung auseinandersetzen, konzentrieren sich auf einige wenige Serien, allen voran Astérix, der zu den sprachlich anspruchsvollsten Comics gerechnet wird. Eine der ersten Artikel, der ausschließlich der Comicübersetzung gewidmet ist, diskutiert die englischen, italienischen und niederländischen Astérix-Versionen und die Übersetzungsproblematik bestimmter Aspekte sprachlicher Komik (Jacqmain/Cole 1970). Die von den Autoren thematisierten Phänomene wie „parodie“, „noms propres“, „rosseries linguistiques“, „plaisanteries“, „jeu de mots" werden dabei definitorisch nicht voneinander abgegrenzt, die zugrundeliegende Komik wird zwar als Übersetzungsschwierigkeit erkannt, jedoch nicht näher analysiert. Dies hätte über die pauschale Erwähnung der kulturellen Einbettung (vgl. Jacqmain/Cole 1970: 4f.) eine genauere 
Auseinandersetzung mit komischen Verfahren, wie plötzlichen Kontextwechseln, dem Spiel mit Doppeldeutigkeiten und Anspielungen, dem Brechen von Erwartungen, die sich aus der kreativen Bisoziation unterschiedlicher Verhaltens- und Wissensbereiche ergeben, notwendig gemacht. Eine solche Analyse würde einen humortheoretischen Rahmen voraussetzen, der von Jacqmain und Cole allerdings zugunsten einer Darstellung praktischer Übersetzungsschwierigkeiten ausgeblendet wird.

Auch Ballweg (1979), der Übersetzungsprobleme der Comicserie Iznogoud von Goscinny/Tabary untersucht, identifiziert in Comics eine „Reihe von Wortwitzelein, Kalauern und ähnlichen Sprachspielerein“ (1979: 1), ohne sie näher zu charakterisieren. Im Gegensatz zu Jacqmain/Cole versucht Ballweg seine Analyse jedoch theoretisch zu fundieren. Er greift dabei auf Saussures Konzept der assoziativen Beziehungen zurück, mit deren Hilfe er auf paradigmatischer und syntagmatischer Ebene die Übersetzung von Namen und Wortspielen näher untersucht. Sein Übersetzungsvergleich dient dabei der besseren Erfassung der Strukturen unterschiedlicher Sprachsysteme. Mit seiner Konzentration auf die langue-Ebene, der Analyse auf Wort- bzw. Satzebene und der völligen Ausblendung nonverbaler Elemente liefert dieser Ansatz allerdings wenig zur Erfassung der Komik, die weder auf Sprache allein noch auf Textstrukturen reduzierbar ist.

Eine fundierte Klassifizierung der zumeist nur pauschal erwähnten Wortspiele und eine zumindest ansatzweise Einbeziehung der bildlichen Elemente in die Untersuchung liefert Grassegger (1985), dessen Studie zur Übersetzung von Wortspielen und Namen, ${ }^{4}$ in Astérix bis heute die wohl fundierteste Arbeit auf diesem Teilbereich der Komik darstellt. Grassegger unterteilt die Wortspiele in Astérix in zwei Gruppen mit je zwei verschiedenen Untergruppen (vgl. 1985: 33f.): In der ersten Gruppe bildet die semantische Motiviertheit das Unterscheidungskriterium für zwei Wortspielarten: Zum einen Sinnspiele, in denen die semantische Ebene für den sprachspielerischen Effekt ausschlaggebend ist, zu denen die wörtliche Interpretation von Redewendungen und das Spiel mit lexikalischer Plurivalenz (Polysemie und Homonymie) sowie jene Fälle von Homophonie, die semantisch akzentuiert sind, gehören. Zum anderen Klangspiele, die semantisch nicht akzentuiert sind und zu denen vor allem nichtsemantische Homophonien, Versprecher und Lautspielerein zu rechnen sind. In dieser zweiten Gruppe werden nach der medialen Komplexität ebenfalls zwei Unterarten unterschieden. Grassegger differenziert hier in Anlehnung an Rothe (1974: 245) zwischen textinternen Sprachspielen, die sich aus dem unmittelbaren oder erweiterten sprachlichen Kontext herleiten lassen und textexternen Wortspielen, an denen auch das Bild beteiligt ist. Für Grassegger kann lediglich das textexterne Wortspiel als comicspezifisch betrachtet werden, während alle anderen Formen auch in rein sprachlichen Texten vorkommen können. Grassegger analysiert zwar im Verlauf der Arbeit einige Wortspiele, die sich aus sprachlich-bildlichen Interaktionen ergeben, aber bereits die Bezeichnung „textexternes“ Wortspiel weist darauf hin, dass das Bild nicht wirklich als integrativer Bestandteil des Gesamttextes betrachtet wird und damit auch keinen zentralen Platz in einem linguistisch motivierten Ansatz hat.

Dieselbe Sprachlastigkeit ist auch in der Studie der arabischen Astérix-Übersetzungen von Hartmann (1982) vorhanden. Im Gegensatz zu Grassegger betont sie allerdings die kulturelle Dimension, die gerade auch beim Verstehen von Komik eine zentrale Rolle spielt. Die kulturelle Dimension als Einflußgröße für die Übersetzung 
von Comics ins Arabische umfasst laut Hartmann insgesamt drei Bereiche: Den erste Faktor bildet die spezifische Sprachsituation im arabischen Raum: Während in der Alltagskommunikation je nach Region unterschiedliche Dialekte verwendet werden, muss schriftlich Fixiertes in der regional übergreifenden Schriftsprache formuliert werden. Das Spiel mit Dialekten und damit zusammenhängend mit nationalen und sozialen Identitäten als eine Quelle der Komik in Astérix entfällt daher in den arabischen Übersetzungen. Laut Hartmann dienen die arabischen Versionen nicht so sehr der Unterhaltung, sondern pädagogischen Zwecken, indem sie die Schüler dazu bringe, das an sich ungeliebte Schriftarabisch zu lesen (vgl. 1982: 1). Als zweiten kulturellen Faktor führt Hartmann die Religion an, der vor allem die Übersetzung von Götternamen insofern beeinflusst, als der Islam als monotheistische Religion, dem Polytheismus intolerant gegenübersteht. Um den arabischen Leserkreis nicht zu brüskieren und auch weil die meisten Götternamen aus Astérix im arabischen Kulturkreis völlig unbekannt sind, werden diese vielfach aus der Übersetzung gestrichen. Den dritten kulturspezifischen Faktor sieht Hartmann im arabischen Nationalgefühl, was wiederum Auswirkungen auf die Übersetzung der parodistischen Darstellung nationaler Klischees und Vorurteile hat, die in Astérix ein wesentliches Mittel zur Erzeugung komischer Wirkungen darstellt. Vor allem die Darstellung der Ägypter in Astérix als dekadent, unzuverlässig und unfähig wurde in der arabischen Übersetzung massiv geändert, um das Nationalgefühl der Leser nicht zu verletzen (vgl. 1982: 24). So wesentlich und richtig die Einbeziehung der kulturellen Dimension in die Debatte der Übersetzung von Komik im Comic ist, so problematisch ist allerdings der Stellenwert, den diese bei Hartmann einnimmt. Hartmann erachtet die von ihr angeführten kulturellen Faktoren als lediglich subjektives Empfinden der Übersetzer (1982: 29), dem sie die objektive Realität der sprachlichen Strukturen gegenüberstellt. Eine solche Trennung in einen sprachlich objektivierbaren und einen kulturell subjektiven Aspekt ist jedoch weder bei die Übersetzung allgemein noch beim Verstehen und Übersetzen von Komik im besonderen vertretbar, da beides nicht als sprachliche, sondern als kulturelle Aktivität verstanden werden muss, die nur auf der Basis eines umfassenden Kulturverstehens erfolgen kann.

Es existieren kaum linguistisch motivierte Arbeiten, die andere Facetten der Komik als das Wortspiel in den Blick nehmen. Zu den raren Ausnahmen zählt Richet (1993), der sich mit Zitaten als Quelle für Komik und ihrer Übersetzung beschäftigt. Damit bezieht sich Richet, allerdings ohne es explizit auszuführen, auf eine wesentliche Technik zur Schaffung von Komik, nämlich intertextuelle Bezüge, zu denen auch Zitate gehören. Richet konzentriert sich auf die parodistische Wirkung von Zitaten in Astérix und unterscheidet dabei zwei Arten: zum einen kulturübergreifende Zitate, wie etwa aus der Bibel, zum anderen kulturspezifische Zitate, die nur in einem Sprach- und Kulturraum vorhanden sind und in der Übersetzung, um ihr komisches Potential entfalten zu können, adaptiert werden müssen. Auch Richet konzentriert sich rein auf die sprachliche Ebene und lässt das Comicspezifische, d.h. die Beteiligung nonverbaler Elemente an der Bedeutungskonstitution völlig außer Acht. Gerade in Astérix wird, wie noch zu zeigen sein wird, Komik allerdings nicht nur mittels sprachlicher Intertextualität, sondern auch durch bildliche Zitate, Anspielungen und Parodien erzielt.

Die Beteiligung des Bildes an der Schaffung von Komik wird zum Teil in semiotisch motivierten Studien diskutiert. Von besonderer Bedeutung ist hierbei die 
Monographie von Stoll (1974) über Astérix, die sich zwar nur am Rande mit der Übersetzung beschäftigt, allerdings für weitere Forschungen von Relevanz sein könnte. Stoll versucht die Mythendekodierung Roland Barthes ${ }^{5}$ auf die Analyse von Comics zu übertragen. Bemerkenswert ist hierbei, dass Stoll nicht nur in der Sprache, sondern auch im Bildmaterial „mythenschöpferische Inszenierungsprozesse“ (1974: 15) freilegt. Nach einer Beschreibung des historisch-kulturellen Umfeldes der Comicserie wendet sich Stoll den spezifischen komischen Strukturen zu, die auf der Parodie des französischen Wertesystems beruhen, wie es durch die 3. und 5. Republik geprägt wurde. In diesem Zusammenhang erfolgt auch eine Ausdehnung der mythologischen Betrachtungsweise auf den bildlichen Teil, in dem kulturell-ideologische sowie gesellschaftliche und politische Werte parodistisch dargestellt werden. In einem kurzen Abschlusskapitel diskutiert Stoll auch die Problematik der Übersetzung und kommt zu dem Schluss, dass sowohl zahllose Wortspiele als auch die Bildparodien außerhalb Frankreichs aufgrund eines anderen Wissens- und Erfahrungshintergrundes nicht nachempfunden werden können und somit unübersetzbar sind (vgl. 1974: 175).

Einen weniger starren Übersetzungsbegriff vertritt Spillner in seinem Artikel zu semiotischen Aspekten der Comicübersetzung (1980). Er fordert die Übersetzungswissenschaft auf, nicht nur Übersetzungsprozeduren für sprachliche Elemente, sondern auch für die nonverbalen Teile von Comics zu entwickeln. Diese Notwendigkeit ergibt sich aus der Beteiligung des Bildes an einer Reihe von Wortspielen, die Spillner anhand einer Reihe von Beispielen untersucht. Dabei unterscheidet er zwei mögliche Relationen zwischen Sprache und Bild: Zum einen eine Identitätsrelation zwischen einer wörtlich genommenen idiomatischen Wendung oder Metapher und der durch das Bild dargestellten situativen Realität und zum anderen eine Kontrastrelation zwischen der wörtlich genommenen Bedeutung einer Redewendung oder Metapher und bestimmten Elementen des bildlichen situativen Rahmens (vgl. 1980: 77f.). Damit beschreitet Spillner einen zweifelsohne vielversprechenden Weg, indem er versucht die Wirkungszusammenhänge zwischen Sprache und Bild bei der Schaffung von Komik näher zu bestimmen.

Auch Zanettin (1998) diskutiert den Text-Bild-Bezug im Comic nicht zuletzt im Hinblick auf die Beteiligung visueller Elemente an der Schaffung von wortspielerischen Effekten. Ähnlich wie Spillner unterscheidet Zanettin zwischen einem Kontrast- und einem Identitätsverhältnis von sprachlicher und bildlicher Aussage. Darüber hinaus unterscheidet er allerdings auch noch zwei grundlegende Wortspieltypen: nämlich solche, die von der Sprache ausgehen und solche, die durch das Bild aktiviert werden (vgl. 1998: 4).

Insgesamt kann festgestellt werden, dass in der Literatur zur Übersetzung von Comics nur eine beschränkte Auswahl der Möglichkeiten zur Erzeugung von Komik als übersetzungsrelevant erkannt und diskutiert wird. Sprache wird im Comic, in Form von Sprechblasentexten vor allem auch dazu genutzt, um soziale Identitäten darzustellen, Beziehungen zu definieren und um kommunikative Situationen zu konstruieren. Zahlreiche Comics, wie etwa Les Bidochons von Binet oder Les frustrés von Brétecher parodieren Personen, die einen bestimmten Sozialtypus verkörpern, und deren Handlungsweisen. Hierbei kommen neben sprachlichen Mitteln, wie etwa Soziolekt, Dialekt und Akzent, auch paraverbale Elemente zum Einsatz, wie etwa Prosodie, Intonation usw., durch die Sprechhandlungen nicht nur dargestellt, son- 
dern auch parodiert werden können. Diese Mittel zur Erzeugung von Komik, die sich durch milieuspezifische sprachliche Darstellungen von Eigenschaften und Verhaltensweisen ergibt, werden in der vorhandenen Literatur so gut wie nicht behandelt. Gleiches gilt für die bildliche Darstellung von Situationen, Ereignissen und Personen, zwar finden sich immer wieder Verweise auf die polysemiotische Beschaffenheit von Comics, diese wird allerdings in den seltensten Fällen auch in den konkreten Analysen ernst genommen. Will man die Komik dieses Genres in seiner Vielfalt und Komplexität analysieren, so ist es zweifelsohne notwendig, die Sprachlastigkeit bestehender Ansätze zu überwinden. Im folgenden sollen daher einige Aspekte visueller Komik untersucht werden, um so den Beitrag bildlicher und typographischer Elemente zur Schaffung eines komischen Potentials zu verdeutlichen.

\section{Von sprachlichen Wortspielen zu nonverbalen Zeichenspielen}

Wie im vorangegangenen Überblick gezeigt wurde, existieren eine Reihe von Untersuchungen zum Wortspiel im Comic, diese konzentrieren sich allerdings vorrangig auf die verbale Ebene und klammern die Möglichkeiten der polysemiotischen Verknüpfung weitgehend aus. Nonverbale Elemente haben jedoch in polysemiotischen Textformen nicht nur die Funktion, den sprachlichen Text zu illustrieren, sie tragen auch wesentlich zur Bedeutungskonstitution bei, sei es in Interaktion mit den sprachlichen Elementen, sei es auch als eigenständiges semiotisches Kommunikationssystem. Diese Tatsache wird nicht nur in comicbezogenen Studien zum Wortspiel, sondern auch in allgemeinen Studien zur Thematik Wortspiel und Übersetzung nur unzureichend berücksichtigt. ${ }^{6}$

Wenn der Einsatz visueller Elemente im Comic auch für eine spielerische Wirkung genutzt werden kann, so bedarf es auch einer näheren Analyse der Beziehungsmöglichkeiten zwischen Sprache und Bild. Die semiotische Komplexität eines Wortspiels hängt dabei von dem Integrationsgrad der beteiligten Zeichen und der Rolle, die Sprache und Bild bei der Herstellung eines wortspielerischen Effekts spielen, ab. Dabei können insgesamt fünf verschiedene Spielarten unterschieden werden:

- $\quad$ rein aus sprachlichen Zeichen bestehende Wortspiele

- mit nonverbalen Zeichen gestützte Wortspiele

- vom semiotischen Verbund abhängige Zeichenspiele

- von verbalen Zeichen gestützte nonverbale Zeichenspiele

- rein aus nonverbalen Elementen bestehende Zeichenspiele

Wie allgemeine Untersuchungen zu visuellen Zeichenspielen gezeigt haben, funktionieren diese nach ähnlichen Prinzipien wie verbale Wortspiele, d.h. sie basieren auf Dichotomieverhältnissen wie z.B. zwischen Form und Inhalt und nützen dabei die Polysemie visueller Zeichen (vgl. Lessard 1991). Für die Nachgestaltung in der Übersetzung ist dabei entscheidend, dass die Beziehungszusammenhänge zwischen verbalen und nonverbalen Zeichen erkannt werden. Welche Faktoren bei der Wiedergabe eines Zeichenspiels berücksichtigt werden müssen, hängt nicht zuletzt von der Rolle $\mathrm{ab}$, die die jeweiligen Bild- und Sprachelemente spielen. Spielt das Bild lediglich eine stützende Rolle und bleibt das Wortspiel auch ohne Bildbezug noch verständlich, so kann die visuell-verbale Relation in der Übersetzung auch von nachgeordneter Bedeutung sein. Ist das Bild jedoch konstitutiv am Aufbau eines Wortspiels beteiligt, 
so sollte möglichst dessen Funktion auch in der Übersetzung - gegebenenfalls durch Veränderung des Bildes, Anmerkungen des Übersetzers oder andere Übersetzungsstrategien - berücksichtigt werden.

Unter nonverbal gestützten Wortspielen ist jene Gruppe zu verstehen, in der das Comicbild zwar einen Bezug zum sprachlichen Wortspiel aufweist, dieser jedoch nicht konstituierend, sondern lediglich unterstützend für die Wirkung des Wortspiels ist. Als Beispiel sei hier eine Sequenz aus Astérix le Gaulois angeführt (Beispiel $1 \mathrm{a}+1 \mathrm{~b})$. Die Römer haben den Druiden und Astérix gefangen genommen und wollen hinter das Geheimnis des Zaubertranks kommen. Der Druide braut jedoch statt der gewünschten Kraftmixtur ein Haarwuchsmittel. Der römische Kommandant verlangt wutentbrannt ein Gegenmittel, Astérix reagiert darauf mit einer Reihe von Redewendungen, in denen Lexeme mit dem semantischen Merkmal "haarig“ vorkommen. Die Unwilligkeit des Druiden, ein Gegenmittel zu brauen, kommentiert Astérix im Französischen mit der Redewendung „Il a un poil dans la main“ (wörtlich: „er hat ein Haar in der Hand“, in übertragenem Sinne: „er hat keine Lust zu arbeiten“) und „il a un cheveux sur la langue aussi“ (wörtlich: „er hat auch ein Haar auf der Zunge“, im übertragenen Sinne: „er lispelt“). Begleitet werden diese Äußerungen durch Gesten, die die Redewendungen wörtlich nehmen: Astérix zeigt im ersten Panel auf seine Hand und im zweiten auf seine Zunge. Die Komik ist im vorliegenden Fall allerdings aufgrund des situativen Kontextes gegeben, die visuellen - gestischen - Elemente unterstützen zwar die Wirkung, sind aber nicht konstitutiv am wortspielerischen Effekt beteiligt. In der deutschen Übersetzung wurde ebenfalls mit einem „haarigen“ Lexem gearbeitet: dieses befindet sich im linken Panel und bereitet gewissermaßen das Wortspiel vor, das dann im rechten Panel durch die Redewendung „Haare auf den Zähnen“ (wörtlich.... auf Englisch, im übertragenen Sinne: „bissig sein“) und das Scheinidiom „Haare auf der Zunge haben“ realisiert wird. Damit wird zumindest eine Übereinstimmung der sprachlichen Äußerung mit der Gestik im zweiten Panel realisiert.
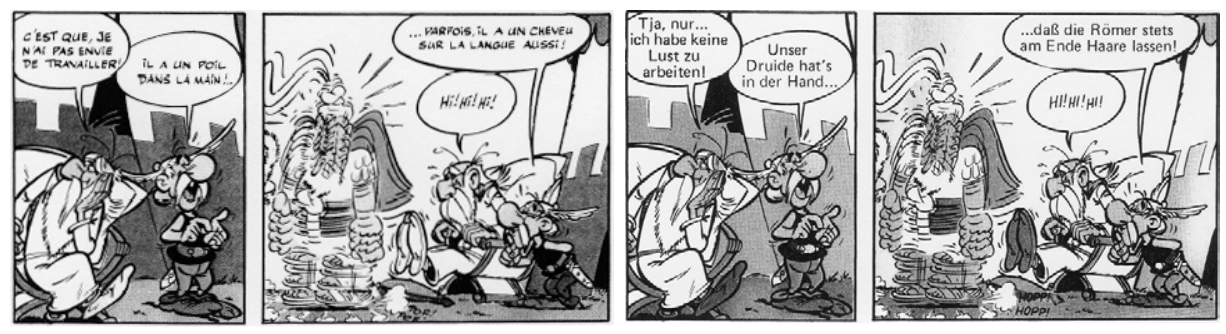

Bei Zeichenspielen, deren Wirkung vom semiotischen Verbund abhängig ist, besteht eine wechselseitige Interaktion zwischen verbalen und nonverbalen Elementen, die konstituierend für den komischen Effekt ist. Als Beispiel sei hier die Beziehung zwischen einer Bildaufschrift und graphischen Zeichen aus Tintin angeführt (Beispiel 2a+2b). Kapitän Haddock hat sich den Kopf an einer Litfasssäule gestoßen, was graphisch durch Sterne dargestellt wird. Dadurch wird ein Identitätsbezug zu der uneigentlichen - wörtlichen - Bedeutung des sprachlichen Textes auf der Säule hergestellt („des informations qui frappent“), mit dem für eine Tageszeitung geworben wird. In der deutschen Übersetzung wurde zwar der Inhalt der sprachli- 

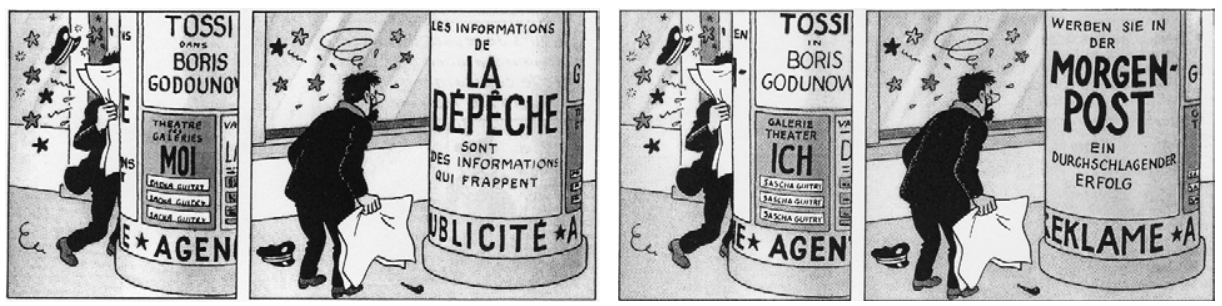

chen Botschaft verändert (während im Original für die Zeitung mit dem Hinweis auf die „umwerfenden“ Nachrichten, die diese bringt, geworben wird, steht in der deutschen Fassung auf der Werbesäule, dass Werbungen in der Zeitung ,Morgenpost' durchschlagenden Erfolg haben) das Zeichenspiel und die zugrundeliegende Identitätsbeziehung zwischen graphischen und sprachlichen Elementen wurde jedoch bewahrt.

Grundsätzlich können jedoch auch die dem Zeichenspiel zugrundeliegenden Beziehungen zwischen verbalen und nonverbalen Elementen in der Übersetzung verändert werden. Ein Beispiel hierfür findet sich in dem bereits zitierten Artikel von Spillner (1980: 78): In der Geschichte Le Bouclier d'Arvergne müssen sich Astérix und Obélix vor den Römern über nacht in einem Kohlehaufen verstecken. Der zeichenspielerische Effekt wird im Französischen durch den Kontrast zwischen der wörtlichen Bedeutung von "passer une nuit blanche“ (wörtlich: „eine weiße Nacht verbringen“, in übertragenem Sinne: „eine schlaflose Nacht verbringen) und dem Bild mit dem schwarzen Kohlehaufen geschaffen. Im Deutschen hingegen wird das Zeichenspiel mittels einer Identitätsbeziehung zwischen der uneigentlichen Bedeutung der Redewendung "Ich seh da in jeder Richtung schwarz" (wörtlich...: in Englisch, in übertragenem Sinne: „ich bin pessimistisch“) und dem schwarzen Kohlehaufen realisiert.

Neben verbalen Wortspielen sind in Comics auch nonverbale Zeichenspiele - mit oder ohne verbale Unterstützung - zu finden. Der Transfer in einen anderen Kulturraum wird vor allem dann problematisch, wenn visuell dargestellte Kulturspezifika am Zeichenspiel beteiligt sind. Ein Beispiel hierfür liefert ein Strip aus Krazy Kat von George Herrimann, in dem ein blindes Schwein abgebildet ist, das - wie aus dem Verlauf der Geschichte hervorgeht - seine Blindheit nur vortäuscht, um anderen Geld abzuknöpfen (Beispiel 3). Der Begriff „blind pig“ bezeichnete in den USA zur Zeit der Prohibition einen Ort, an dem man illegal Alkohol konsumieren konnte. Die Komik resultiert aus der Übertragung der uneigentlichen Bedeutung in einen Bild-

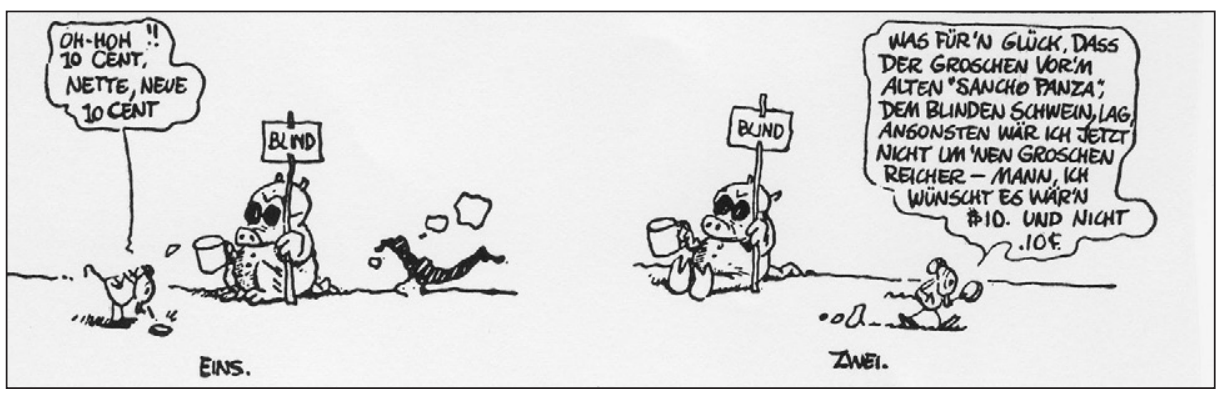


code, der noch durch das auf einer Tafel geschriebene „blind“ verbal gestützt wird. In der deutschen Übersetzung wurde das Bild belassen, der Übersetzer fügte lediglich eine Erklärung am Ende des Bandes bei, in dem das Zeichenspiel für den deutschsprachigen Leser erklärt wird.

\section{Bild und Komik}

Die Tatsache, dass bildliche Elemente lange Zeit kaum als übersetzungsrelevant erachtet wurden liegt zum einen in der Entwicklungsgeschichte des Faches begründet. Die Einengung des Objektbereichs auf die sprachliche Ebene führte dazu, dass polysemiotische Texte, wie etwa Film, Werbung, Theater oder eben Comics erst mit der Etablierung der Translationswissenschaft als eigener Disziplin in den 80er Jahren verstärkt Beachtung fanden. Zum anderen scheint in der Übersetzungswissenschaft noch immer der Glaube an eine universell verständliche Bildsprache zu existieren. So meint etwa Rabadán, Bilder stellten einen universellen Code dar, der demnach auch nicht übersetzt werden müsse: „El dibujo, come la fotografía publicitaria o la imagen cinematográfica, constituye un lenguaje universal y por lo tanto no traducible." (1991: 154) Der Glaube, es gäbe ein visuelles Esperanto, geht auf die Ansicht zurück, zwischen Abgebildetem und Wirklichkeit bestehe eine unmittelbare Ähnlichkeit, die Bilder von den - arbiträren - sprachlichen Zeichen unterscheiden würden. Nicht zuletzt Eco hat jedoch deutlich gemacht, dass Bilder einen durch Konventionen geregelten Code aufweisen und Bildkonventionen dabei auch kulturell determiniert sein können (vgl. 1972: 202). Dies bedeutet auch, dass die visuelle Darstellung von Gegenständen, Gesten, mimischen Ausdrücken usw. nur dann in ihrer Bedeutung erkannt wird, wenn die Kultur diese Elemente auch definiert hat (vgl. Eco 1987: 65).

Comics weisen einen besonders hohen Grad an Konventionalisierung auf (vgl. Kloepfer 1976: 43), sowohl was die Anordnung der einzelnen Panels, also die Makrostruktur, als auch auf mikrostruktureller Ebene die bildliche Darstellung innerhalb der Panels betrifft. Darüber hinaus kann die Darstellung von Realia oder auch kulturspezifischen Verhaltensweisen zu Verstehensproblemen bei der Übersetzung führen. Dass dies auch Auswirkungen auf die Wiedergabe von Komik haben kann, sei anhand einiger Beispiele dargestellt.

Wie diskursanalytische Untersuchungen von Humor gezeigt haben, haben auch nonverbale Aspekte, wie etwa Mimik und Gestik oft entscheidenden Anteil daran, ob eine Äußerung als komisch oder humorvoll erachtet wird. Diese enge Vernetzung von verbalen und nonverbalen Elementen kommt auch bei der Darstellung von Handlungen in Comics zum Tragen, in denen die Dialoge aufs engste mit der visuellen Darstellung der Figuren verbunden sind. In der Geschichte L'homme aux 7 douleurs aus Les frustrés von Claire Brétecher etwa, in der es um einen Hypochonder geht, der seine Lebensgefährtin regelmäßig mit seinen eingebildeten Krankheiten behelligt, resultiert die Komik aus dem Spannungsverhältnis zwischen dem gelangweilt-routinierten Umgang der Frau mit den Ängsten des Hypochonders und der Wahrnehmung dieses Verhaltens durch den Hypochonder als ernsthaftes Eingehen auf seine Beschwerden (Beispiel 4a+4b). Dabei kommen sowohl sprachliche als auch gestische Mittel zum Einsatz. Zu letzterem ist auch die formelle Schwurgeste zu zählen, mit der die Frau ihrem Lebensgefährten ohne zu zögern versichert, notfalls 

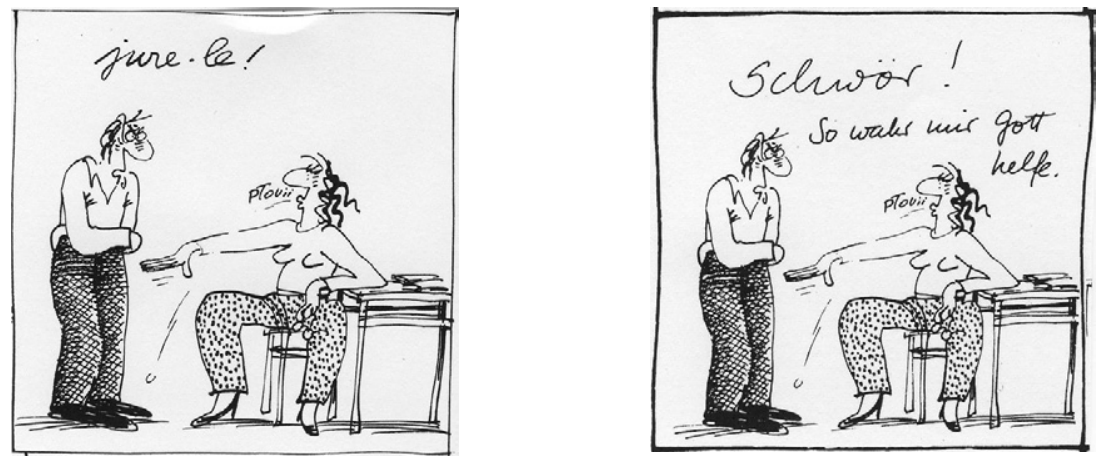

eigenhändig lebenserhaltende Schläuche, an denen er vielleicht zwecks Lebensverlängerung angeschlossen wird, zu entfernen. Im Gegensatz zu Frankreich wird allerdings ein Schwur im deutschsprachigen Raum nicht gestisch und mimisch mit horizontal ausgestreckter Hand und Auf-den-Boden-spucken dargestellt, sondern mit erhobener Hand, wobei Zeige- und Mittelfinger, die zuvor mit der Zunge berührt worden sind, ausgestreckt werden. Durch die Fremdheit der Geste für den deutschen Leser, der darin nicht unmittelbar einen Schwur erkennt, wird auch der komische Kontrast in der Verhaltensweise der Frau reduziert.

Allerdings stellt die Adaptierung solcher kulturspezifischer bildlicher Elemente nicht immer die Lösung für die Wahrung des komischen Effekts dar. So wurde in der Serie Peanuts von Charles Schulz der ovale american football für die deutsche Übersetzung in der Zeitschrift Stern durch einen runden europäischen Fußball ersetzt. Der Fußball ist Teil eines running gag, der darin besteht, dass Lucy den Ball hält und wenn Charlie Brown versucht, den Ball zu treffen, diesen wegzieht, so dass Charlie durch die Luft segelt und unsanft auf dem Boden landet. Die gestische Handlung des Ballhaltens macht dabei in der amerikanischen Fassung durchaus Sinn, da beim „american football“ ein ovaler Ball verwendet wird, der festgehalten wird, damit er nicht umkippt. In der deutschen Fassung mit einem runden Ball ist diese Handlung jedoch in den Fußballregeln nicht vorgesehen, das Halten des Balls wirkt eher unmotiviert bzw. scheint nur dazu zu dienen, ihn wegzuziehen, wodurch der Gag an Wirkung verliert.

Wesentlichen Anteil an der Schaffung von Komik durch visuelle Mittel haben auch intertextuelle Bezüge. Zu diesen zählen laut Genette (1982) neben Zitaten, auch Plagiate, textuelle Anspielungen und vor allem Parodien. Unter einer Parodie sei dabei die Imitation von Strukturen des Quellentextes, die mit einer neuen Funktion versehen werden, verstanden (vgl. Kotoff 1996: 264). Das Verstehen von Parodien ist dabei kulturgebunden. In Comics finden sich neben sprachlichen Parodien immer wieder auch Bildparodien, wobei vor allem auf beliebte Sujets bzw. bekannte Gemälde referiert wird. So etwa beziehen sich Goscinny und Uderzo in der Episode Astérix en Corse auf parodistische Weise auf historische Gemälde, wie sie unter anderem von Vernet, Gérard und de Roehn von der Schlacht bei Austérlitz 1805 angefertigt wurden, sowie die nach diesen Vorbildern angefertigten ,images d'Epinal“. Zentrales Element stellt dabei die aufgehende Sonne dar, durch die die "Grande Armée“ Napoléons einen entscheidenden Sichtvorteil erhielt und so die Schlacht für sich entscheiden konnte. Die Bildparodie, die das Geschehen nach Korsika verlegt, wird 
durch eine sprachliche Anspielung sowie ein sprachliches Klangspiel ergänzt, die den Bezug zu Austerlitz auch verbal deutlich machen. Einerseits heisst der Anführer der korsischen Rebellen Osterlix, das phonetisch praktisch mit dem französischen Austerlitz identisch ist. Andererseits spricht der Fremdenführer im unteren rechten Bild vom „sommeil d'Osterlix“, eine spielerische Abwandlung des französischen „soleil d'Austerlitz“, die auch noch graphisch durch eine stilisierte gezackte Darstellung der Sonne im Hintergrund versinnbildlicht wird. In der deutschen Übersetzung geht aufgrund des fehlenden kulturellen Hintergrundwissens was die bildlichen Darstellungstraditionen dieses Ereignisses betrifft, die parodistische Wirkung dieser Sequenz verloren.

Ähnlich verhält es sich auch mit parodistischen Anspielung auf ein berühmtes Gemälde von Géricault in der Geschichte Coke en stock von Hergé (Beispiel 5a+5b). Tintin und sein Kompagnon Haddock sowie ein Pilot names Szut sitzen als Schiffbrüchige auf einem Floss. Als ein Dampfer naht, der Rettung verspricht, hüpft Haddock vor Freude in die Luft, das Floss zerbricht und er landet im Meer. Als er wieder auftaucht, hat er eine Qualle auf dem Kopf, was Tintin mit „Vous voulez donc à tout prix que ce soit réellement le Radeau de la Méduse“ kommentiert. Mit „Radeau de la Méduse“ wird auf des gleichnamige Gemälde von Géricault verwiesen, auf dem die Überlebenden einer Fregatte namens „Méduse“ auf einem Floß abgebildet sind. Der parodistische Verweis wird dabei gleichzeitig mit einem zeichenspielerischen Effekt verbunden, indem die bildlich dargestellte Qualle auf Haddocks Kopf (franz.: méduse) mit dem nach der mythologischen Figur der Medusa benannten Boot gleichgesetzt wird. In der deutschen Übersetzung wird weder das visuell gestützte Zeichenspiel noch der parodistische Bezug zu Géricaults Gemälde nachvollzogen, statt dessen fragt Tintin, ob Haddock nach seinem unfreiwilligem Untertauchen im Meer noch immer Durst habe.
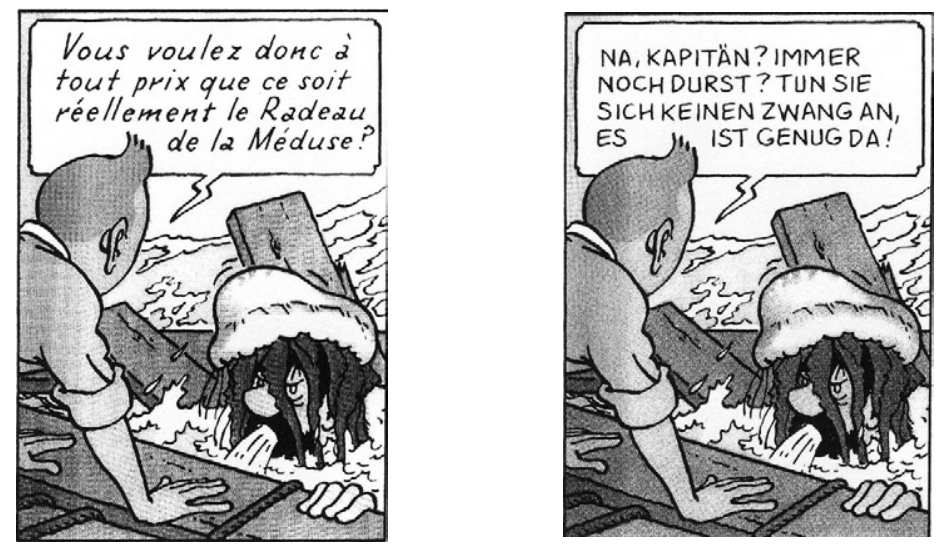

Neben Bildparodien finden sich im Comic auch immer wieder Anspielungen auf Elemente der visuellen Kultur. Während bei Genette Anspielungen auf textuellen Bezügen basieren müssen, fasst Wilss (1989: 45) Anspielungen weiter und versteht darunter auch jene Phänomene, die auf kulturspezifische Wissensbestände referieren. Solche werden vor allem bei jenen Comics vorausgesetzt, in denen die erzählten Geschichten eng mit dem sozialen, politischen, ideologischen etc. Hintergrund einer 
Kultur verknüpft sind. Als Beispiel sei hierfür die Figur des Premierministers im französischen Comic Monsieur le Ministre angeführt. Die gezeichnete Figur ist, nicht zuletzt auch aufgrund der an politische Zeitungskarikaturen erinnernde physische Darstellung, als Anspielung auf Jacques Chirac zu verstehen, der in den 80er Jahren als Premierminister unter dem sozialistischen Präsidenten François Mitterand fungierte und sich mehrmals vergeblich um das Präsidentenamt beworben hatte, bevor er 1995 in den Elysée-Palast einzog. Die säuerliche Miene des Premierministers spielt mit dem Wissen des französischen Lesers, dass Chirac eigentlich lieber das Präsidentenamt übernommen hätte. In der deutschen Übersetzung entfällt mit dem entsprechenden kulturellen Hintergrundwissen auch die mit dieser Anspielung intendierte komische Wirkung Beispiel 6).

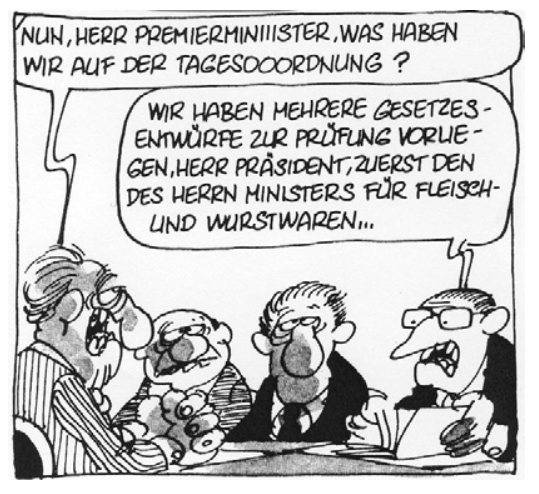

Doch auch Anspielungen auf kulturübergreifende Phänomene, wie zum Beispiel bestimmte Märchen, können in der Übersetzung zum Problem werden, wenn die damit verbundene visuelle Darstellung Unterschiede aufweist. Ein solcher Fall liegt in der bereits zitierten Geschichte Coke en stock von Hergé vor (Beispiel 7). Als Tintin und Haddock nach Hause kommen, stürzt Milou, der Hund von Tintin diesen mit lautem Heulen entgegen. Der Grund ist die seltsame Verkleidung mit einer roten Haube und einem rosa Umhang, die ihm Abdallah, der Sohn eines mit Tintin und Haddock befreundeten Emirs, der vorrübergehend bei Haddock wohnt, verpasst hat und die Milou offensichtlich als erniedrigend erachtet. Diese Aufmachung spielt auf die Figur des Rotkäppchens an, die in französischen Märchenbüchern mit eben dieser
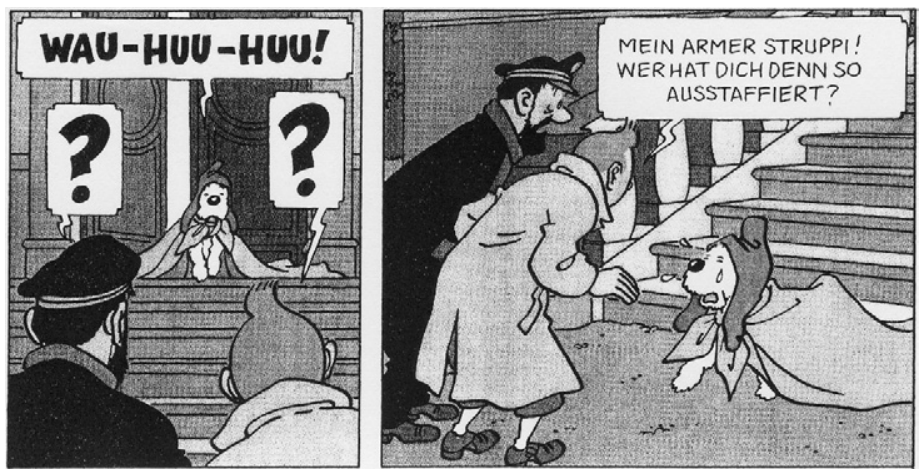
typischen Haube und einem solchen Umhang traditionell abgebildet wird. Die Komik resultiert aus dem Kontrast zwischen der naiven Figur des Rotkäppchens aus dem Märchen von Perrault, auf die hier angespielt wird, und dem an sich als äußerst klug und detektivisch begabten Hundes Milou, den Hergé mit durchaus menschlichen Verhaltensweisen zeichnet, wie etwa im vorliegenden Fall, in dem der Hund vor Scham über die lächerliche Verkleidung weint. Im deutschsprachigen Raum wird Rotkäppchen jedoch kleidungsmäßig anders dargestellt, nämlich mit einer roten Kappe und einem Mantel, wodurch der Leser den komischen Kontrast zwischen Märchen- und Comicfigur nicht herstellen kann.

Das visuelle Repertoire im Comic umfasst jedoch nicht nur ikonische Zeichen, wie die Darstellung von Figuren und Gegenständen, sondern auch graphische und typographische Elemente, die ebenfalls an der Erzeugung von Komik beteiligt sein können. Vor allem die Typographie im Comic hat sich zu einem eigenständigen Ausdrucksmedium entwickelt, durch das eine Reihe von Informationen vermittelt werden kann. Durch die Schriftart werden in Astérix die verschiedenen Nationalitäten typographisch dargestellt, z.B. die Ägypter durch Hieroglyphen, die Goten durch Frakturschrift etc., die Proportionsverhältnisse, also die Höhe und Breite der Buchstaben, das Laufbild, also der geradlinige, gebogene, fluktuierende Verlauf der Schrift, das Duktusverhältnis, d.h. der Abstand der Buchstaben zueinander bzw. die Neigung der Buchstaben zur Grundlinie, sowie die Umrissmodulationen, also die Konturen der Buchstaben, dienen dazu Lautstärke, Tonhöhe und Tondauer von Äußerungen und Geräuschen darzustellen. Die Farbigkeit schließlich kann dazu eingesetzt werden, durch damit verbundene Symbole zusätzliche Informationen z.B. über die emotionale Befindlichkeit von Figuren Aussagen zu machen.

Diese typographischen Elemente werden sowohl bei den Äußerungen der Comicfiguren eingesetzt als auch bei der Darstellung von Geräuschen, den sogenannten Onomatopoien. In der Übersetzung wurde und wird zum Teil auch heute noch die typographische Dimension und ihr kommunikatives Potential vernachlässigt. Die Visualisierung von akustischen Aspekten des Sprechakts kann jedoch durchaus auch Komik erzeugen. Als Beispiel sei hier eine Sequenz aus Astérix le Gaulois und der kroatischen Übersetzung angeführt (Beispiel 8a+8b). ${ }^{7}$ Astérix neckt einen Römer, indem er ihm heftig an seinem Bart zieht und dabei ein Kinderlied singt, in dem das Lexem „Bart“ vorkommt („Je te tiens par la barbichette). Die heftige Kopfbewegung, die bei dem Römer durch das Ziehen am Bart verursacht wird, wird
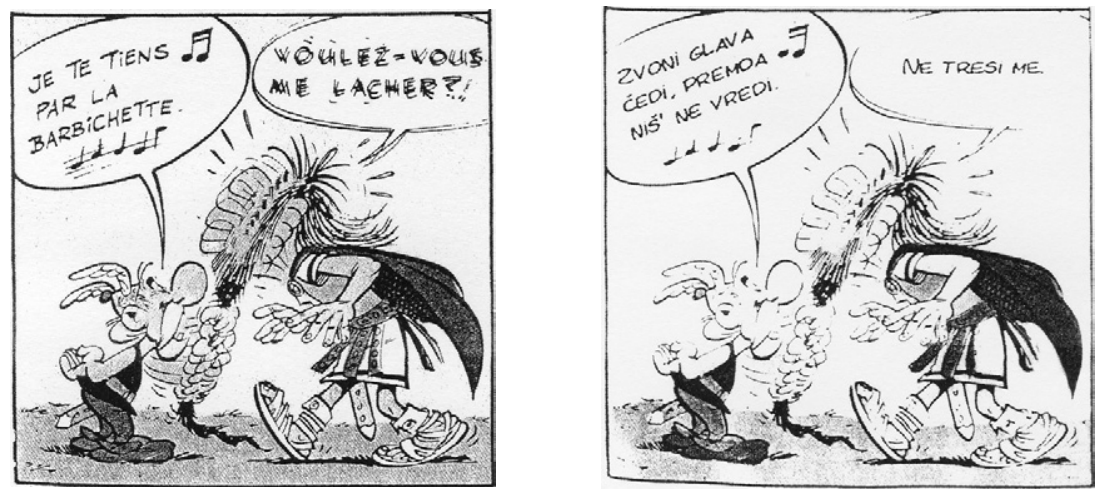
durch das Bild verdeutlicht, das dadurch verursachte Zittern der Stimme wird durch die Doppelung des Laufbildes typographisch visualisiert. Dieser letztere komische Aspekt wurde in der kroatischen Übersetzung ignoriert, durch die Typographie wird hier eine normale Stimmführung suggeriert.

\section{Komik und Onomatopoien}

Abschließend soll noch kurz auf den Zusammenhang von Komik und Onomatopoien eingegangen werden. Auf das komische Potential dieses gerade in Comics häufig vorkommenden Sprachvorkommens hat bereits Nash (1985: 126ff.) verwiesen, wobei er betont, dass Komik und Witz nicht per se gegeben sind, sondern erst in humoristischen Zusammenhängen als solche empfunden werden. Diese müssen vom Leser allerdings erkannt werden, was nicht immer von vornherein gegeben ist. Onomatopoien werden häufig mit dem Hinweis unübersetzt gelassen, sie seien aufgrund ihres lautnachahmenden Charakters ohnehin international verständlich (vgl. Havlik 1981: 13) und müssten daher nicht übersetzt werden. Die Verschriftlichung von Geräuschen erfolgt allerdings immer durch die mehr oder weniger willkürliche Auswahl bestimmter Lautmerkmale, so dass niemals die gesamte Lautfülle in Schriftform wiedergegeben wird, sondern immer auch ein gewisser Grad an Arbitrarität bestehen bleibt. Onomatopoien können auch über ihre Geräuschfunktion hinaus Informationen transportieren, die in entsprechenden Zusammenhängen als komisch empfunden werden. Sind diese Informationen kulturspezifisch geprägt, so stellen sie auch ein Übersetzungsproblem dar. Wenn zum Beispiel Uderzo im jüngsten Abenteuer Astérix et Latraviata die Schläge, die Astérix und Obélix den Römern verpassen, mit den Lauten „tchac! tchic! tchraac! vertont, so stellt dies eine leicht verfremdete phonetische Wiedergabe des Namens des französischen Staatspräsidenten Jacques Chirac dar. In der deutschen Übersetzung wurde diese Onomatopoie phonetisch leicht angepasst und auch eine weitere politische Person in onomatopoietischer Form eingefügt, nämlich Joschka Fischer, der damalige Aussenminister Deutschlands (Beispiel 9a+9b).
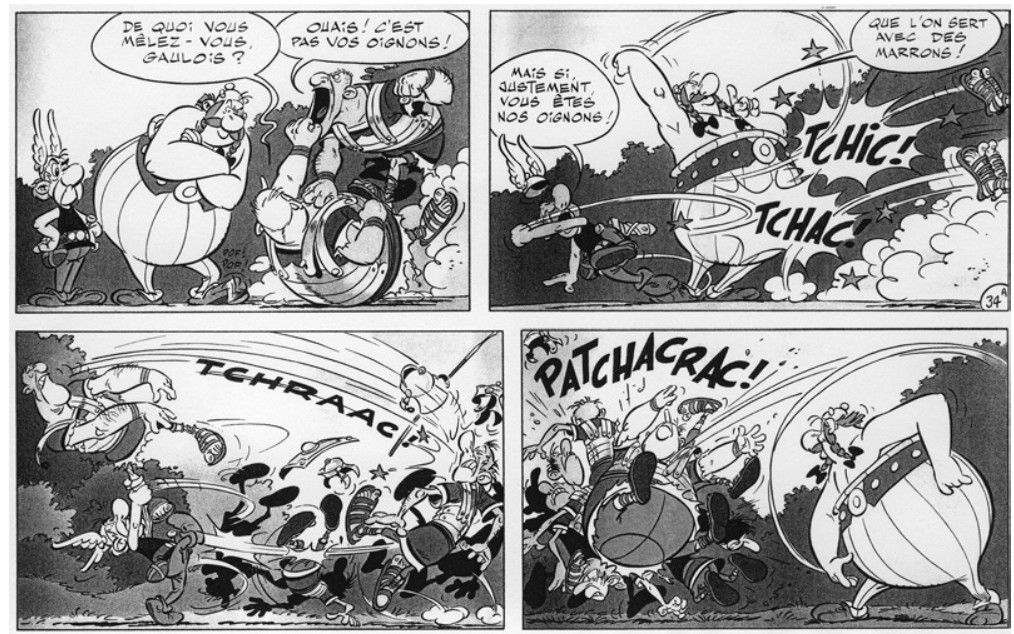

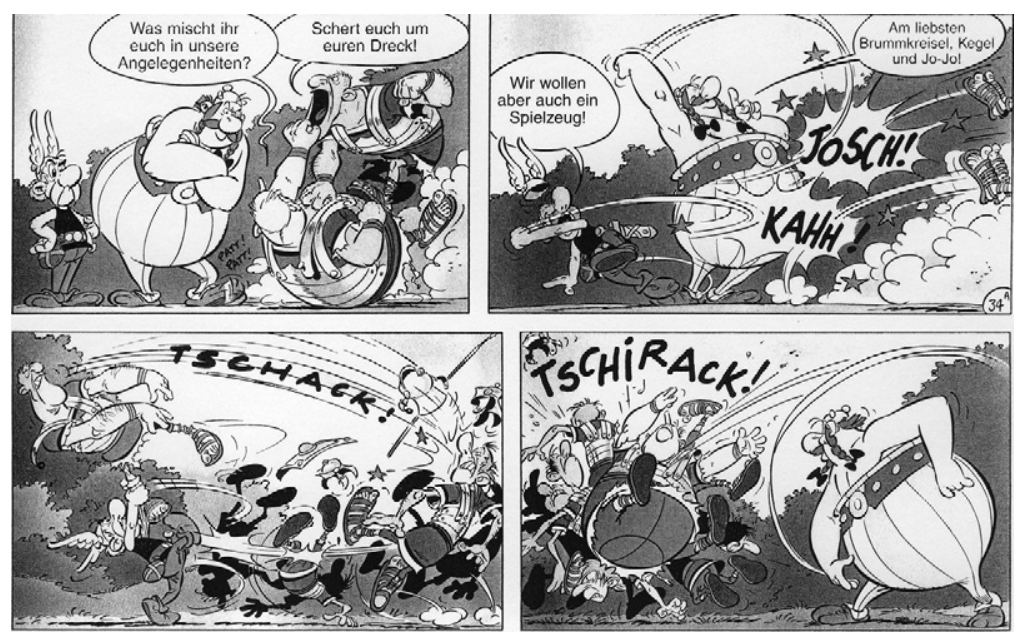

\section{Zusammenfassung}

Comics stellen ein komplexes semiotisches System dar. Die verschiedenen verbalen und nonverbalen Elemente dieser Gattung können grundsätzlich alle auch zur Erzeugung von Humor und Komik eingesetzt werden. Die Palette der Kommunikationsmittel im Comic reicht dabei von den sprachlichen über die bildlichen bis hin $\mathrm{zu}$ den typographischen Zeichen. In humortheoretischen Studien allgemein aber auch in der Übersetzungswissenschaft im besonderen besteht bis heute eine eher sprachlastige Untersuchungsperspektive, die Bedingungen und Funktionsweisen nonverbalen Humors weitgehend ausklammern. Besonders was Comics betrifft, wurde in übersetzungswissenschaftlichen Arbeiten bisher nur ein sehr kleiner Teil der verschiedenen Techniken, die in Comics zur Erzeugung von Humor und Komik eingesetzt werden können, analysiert. Dabei standen in den meisten Arbeiten zur Comicübersetzung vor allem sprachliche Wortspiele im Mittelpunkt. Andere Faktoren sprachlicher Komik und sprachlichen Humors, wie z.B. soziale Typisierung, Ironie, Spiel mit Gattungsverweisen usw. wurden so gut wie gar nicht behandelt. Humor und Komik entstehen jedoch nicht nur auf sprachlicher Ebene durch das Schaffen neuer kommunikativer Zusammenhänge, die nicht im Rahmen der normierten Verständigung stehen, auch paraverbale und nonverbale Zeichensysteme schaffen durch Bisoziation, das Brechen von kommunikativen Erwartungen und interpretative Vielfalt komische und humorvolle Zusammenhänge. Die diesbezügliche Rolle des Bildes, sowohl was Komik im allgemeinen als auch das Wortspiel im besonderen betrifft wurde in den einschlägigen Arbeiten allerdings bisher weitgehend vernachlässigt. In diesem Beitrag habe ich versucht, neben einer Typologie verschiedener Zeichenspiele auch Techniken visueller Komik aufzuzeigen. Hierbei wurden vor allem die verschiedenen Formen intertextueller Beziehungen, von der Parodie bis hin zur Anspielung als wesentliches Mittel zur Erzeugung von visueller Komik definiert. Dabei wurde auch deutlich, dass das Verstehen bildlich dargestellter komischer Zusammenhänge sehr stark vom kulturellen Wissenshintergrund des Rezipienten abhängig ist. 
Die mit der Loslösung von den philologischen Ausgangsdisziplinen eingeleitete Ausdehnung des übersetzungswissenschaftlichen Untersuchungsinteresses auf neue Fragestellungen und neue, Bereiche, wie etwa polysemiotische Texte wie Film, Werbung, Oper und eben auch Comics, hat wesentlich dazu beigetragen, die „success story“ (Bassnett/Lefevere 1990: ix) des Faches in den 80er und 90er Jahren zu schreiben. Allerdings sind die meisten Arbeiten im Bereich semiotisch komplexer Texte bis heute von einer - durchaus auch als Erbe der auch über die Sprache hinaus Analysemethoden und philologischen Ausgangsdisziplinen anzusehenden - Konzentration auf die sprachliche Ebene gekennzeichnet. Nicht zuletzt aufgrund der wachsenden Bedeutung der visuellen Dimension von Texten sollte es aber auch eine Aufgabe der Übersetzungswissenschaft sein, Analyseinstrumentarien und Übersetzungsprozeduren für nonverbale Elemente zu entwickeln, um so die Übersetzung semiotisch komplexer Texte als ganzheitliches Phänomen zu begreifen.

\section{NOTES}

1. Zu einer ausführlichen Darstellung der Geschichte der Comics vgl. Waugh (1947) für den USamerikanischen Raum, Moliterni (1972) für den frankophonen Raum und Dolle-Weinkauff (1990) für den deutschsprachigen Raum.

2. Zur einer übersetzungsrelevanten Anatomie von Comics vgl. Kaindl (1999a).

3. Zur Geschichte und Definition des Humorbegriffs vgl. u.a. Ueding (1992).

4. Die Problematik der Namensübersetzung wird u.a. auch ausführlich von Embleton (1991) diskutiert, allerdings geht es ihr dabei nicht so sehr um das komische Potential, dem Spiel mit den Namen, sondern um eine deskriptive Darstellung der verschiedenen Übersetzungsstrategien.

5. Laut Barthes kann jedes Objekt grundsätzlich zum Mythos werden, Voraussetzung dafür ist „un usage social qui s'ajoute à la pure matière“ (1957: 216, Hervorhebung im Original).

6. So erwähnen zwar z.B. Heibert (1993: 95) in seiner allgemeinen Studie zur Wortspielbübersetzung und Gottlieb (1997) im Hinblick auf die Filmübersetzung die Möglichkeit polysemiotischer Verknüpfungen, in der Folge werden allerdings nur sprachliche Wortspiele diskutiert.

7. Für eine ausführliche Analyse der kroatischen Übersetzung vgl. Kadric/Kaindl (1997).

\section{REFERENCES}

\section{Literatur}

\section{Primärliteratur}

Binet (1989): Monsieur le Ministre, Paris, Editions Audie. Dt. Übersetzung (1990): Herr Minister, Nürnberg, alpha-comics.

Brétecher, C. (1979): Les frustrés 4, Barcelona, Printer Industria Gráfica. Dt. Übersetzung (1989): Die Frustrierten 4, Reinbek b, Hamburg, Rowohlt.

Goscinny, R. et A. Uderzo (1961): Une aventure d'Astérix le Gaulois, Paris, Dargaud. Dt. Übersetzung (1968): Astérix der Gallier, Stuttgart, Ehapa. Kroat. Übersetzung (1992): Asterix Gal, Zagreb, Izvori.

Goscinny, R. et A. Uderzo (1963): Astérix et les Goths, Paris, Dargaud. Dt. Übersetzung I (1965): Siggi und die Ostgoten, in Lupo modern 37. German Translation II (1971): Asterix und die Goten, Stuttgart, Ehapa.

Goscinny, R. et A. Uderzo (1967): Astérix Légionnaire, Paris, Dargaud. Dt. Übersetzung (1971): Asterix als Legionär, Stuttgart, Ehapa.

Goscinny, R. et A. Uderzo (1968): Le bouclier d'Arverne, Paris, Dargaud. Dt. Übersetzung. (1972): Asterix und der Avernerschild, Stuttgart, Ehapa.

Goscinny, R. et A. Uderzo (1970): Astérix en Corse, Paris, Dargaud. Dt. Übersetzung (1975): Asterix auf Korsika, Stuttgart, Ehapa. 
Hergé (1947): Tintin - Le Trésor de Rackham le Rouge, Paris et Tournai, Castermann. Dt. Übersetzung (1971): Tim und Struppi - Der Schatz Rakham des Roten, Hamburg, Carlsen. Hergé (1958): Tintin - Coke en Stock, Paris et Tournai, Castermann. Dt. Übersetzung (1970): Tim und Struppi - Kohle an Bord, Hamburg, Carlsen.

Herriman, G. (1992): Krazy Kat, Vol 2 (Übersetzung von Harald Havas), Wien, Comicforum. Schulz, Ch. M. (1981): 'Die Peanuts', in Stern 47.

Uderzo, A. (2001): Astérix et Latraviata, Paris, Les Editions Albert René. Dt. Übersetzung. (2001): Asterix und Latraviata, Berlin, Egmont Ehapa.

\section{Sekundärliteratur}

Attardo, S. (2001): Humorous Texts: A semantic and pragmatic analysis, Berlin, Mouton de Gruyter.

Attardo, S. (1994): Linguistic Theories of Humor, Berlin and New York, Mouton de Gruyter.

Attardo, S. and V. RAskin (1991): "Script Theory Revis(it)ed: Joke similarity and joke representation model”, Humor 4-3/4, pp. 293-347.

Ballweg, J. (1979): "Iznogoud, Dilat Lahrat, Saussures Assoziative Relationen und das Problem der Übersetzung”, in Bergerfurth, W. (Hg.), Festschrift für Rupprecht Rohr zum 60. Geburtstag, Heidelberg, Groos, pp. 1-12.

Barthes, R. (1957): Mythologies, Paris, Seuil.

Cецотті, N. (1997): «Langue et image en présence: des espaces langagiers pluriels comme moment de réflexion pour la traductologie contemporaine», in L'histoire et les théories de la traduction, Bern and Genève, ASTITI/ETI, pp. 487-503.

Delabastita, D. (1993): There's a double tongue. An investigation into the translation of Shakespeare's wordplay, with special reference to "Hamlet", Amsterdam/Atlanta, Rodopi.

Dolle-Weinkauff, B. (1990): Comics. Geschichte einer populären Literaturform in Deutschland seit 1945, Weinheim and Basel, Beltz.

Eco, U. (1972): Einführung in die Semiotik, München, Fink.

Eco, U. (1986): “The Comic and the Rule”, in Eco, U., Travels in Hyperreality, New York, Harcourt Brace, pp. 269-279.

Eco, U. (1987): Semiotik. Entwurf einer Theorie der Zeichen, München, Fink.

Embleton, S. (1991): "Names and Their Substitutes. Onomastic Observations on Astérix and Its Tranlsations", Target 3-2, pp. 175-206.

Genette, G. (1982): Palimpsestes: La littérature au second degré, Paris, Seuil.

Grassegger, H. (1985): Sprachspiel und Übersetzung. Eine Studie anhand der Comic-Serie Astérix, Tübingen, Stauffenburg.

Hartmann, R. (1982): “Betrachtungen zur arabischen Version von Astérix. Ein Übersetzungsvergleich”, Linguistische Berichte 81, pp. 1-31.

Havlik, E. J. (1981): Lexikon der Onomatopoien. Die lautimitierenden Wörter im Comic, Frankfurt a. M., Fricke.

Hunoltstein, B. (1996): Manipulative Eingriffe in die deutschen Disney-Comics, Köln, Der Donaldist (Sonderheft 34).

Jacqmain, M. et H. Cole (1970): «Astérix à la conquête de l'Europe», Babel 16-1, pp. 4-12.

Kadric, M. und K. KaindL (1997): "Astérix - vom Gallier zum Tschetnikjäger: Zur Problematik von Massenkommunikation und übersetzerischer Ethik", in SNELL-Hornby, M., Jettmaroví, Z. and K. KAINDL (Hg.), Translation as Intercultural Communication, Selected Papers from the EST Congress - Prague 1995, Amsterdam and Philadelphia, John Benjamins, pp. 135-146.

KaIndL, K. (1999a): “Thump, Whizz, Poom: A Framework for the Study of Comics under Translation”, Target 11-2, pp. 263-288.

KaIndL, K. (1999b): “Warum sind alle Japaner Linkshänder? Zum Transfer von Bildern in der Übersetzung von Comics”, TextConText 13-1, pp. 1-24.

KAINDL, K. (2004): Übersetzungswissenschaft im interdisziplinären Dialog. Am Beispiel der Comicübersetzung, Tübingen, Stauffenburg. 
Kerbrat-Orechioni, C. (1981): «Les usages comiques de l'analogie», Folia Linguistica 15-1/2, pp. 163-183

Kloepfer, R. (1976): "Komplementarität von Sprache und Bild (Am Beispiel von Comic, Karikatur und Reklame)", Sprache im technischen Zeitalter 57, pp. 42-56.

Koestler, A. (1964): The Act of Creation, London, Hutchinson.

Котоғғ, Н. (1996): Spass verstehen. Zur Pragmatik von konversationellem Humor, Wien, unpubl. Habilitation.

LANnö̈, P. (1991): Asterix in Britain, Marseille, Bédésup.

Lessard, D. (1991): Calembours et dessins d'humour. Semiotica 85/1-2, pp. 73-89.

LefÈvre, P. and C. Dierick (1998): Forging a New Medium: The comic strip in the nineteenth century, Bruxelles, VUB Univ. Press.

Moliterni, C. (1972) : Histoire de la bande dessinée d'expression française, Paris, SERG.

Mounin, G. (1967): Die Übersetzung. Geschichte, Theorie, Anwendung (aus dem Ital. übersetzt von Harro Stammerjohann), München, Nymphenburg.

Nash, W. (1985): The Language of Humour. Style and Technique in Comic Discourse, London, Longman.

RABADÁN, R. (1991): Equivalencia y traducción, León, Universidad de León.

RAskin, V. (1985): Semantic Mechanisms of Humour, Dordrecht, D. Reidel.

Richet, B. (1993): «Quelques réflexions sur la traduction des références culturelles. Les citations littéraires dans Astérix", in BALlaRD, M. (dir.) La traduction à l'Université. Recherche et propositions didactiques, Lille, Presses Universitaires de Lille, pp. 199-122.

Roтнe, W. (1974): “Astérix und das Spiel mit der Sprache”, Die Neueren Sprachen 23, pp. 241261.

Schmidt, S. J. (1976): “Komik im Beschreibungsmodell kommunikativer Handlungsspiele”, in Preisendanz, W. und R. Warning (Hg), Das Komische, München, Fink, pp. 165-191.

Schmitт, P. A. (1997): "Comics und Cartoons: (kein) Gegenstand der Übersetzungswissenschaf?", in Drescher, H. W. (Hg), Transfer: Übersetzen - Dolmetschen - Interkulturalität. 50 Jahre Fachbereich Angewandte Sprach- und Kulturwissenschaft der Johannes GutenbergUniversität Mainz in Germersheim, Frankfurt a. M.: Peter Lang, pp. 619-662.

Schwarz, A. (1989): Comics übersetzen - besonders ins Deutsche und besonders in der Schweiz, Lausanne, CTL (Travaux du centre de traduction littéraire 4).

SpILlner, B. (1980): "Semiotische Aspekte der Übersetzung von Comic-Texten", in Wilss, W. (Hg), Semiotik und Übersetzen, Tübingen, Narr, pp. 73-85.

Stoll, A. (1974): Astérix. Das Trivialepos Frankreichs. Die Bild- und Sprachartistik eines Bestseller-Comics, Köln, M. DuMont Schauberg.

Ueding, G. (1992): "Ansätze zu einer Textsortensemantik am Beispiel des Witzes", in Detering, K. et al. (Hg), Sprache erkennen und verstehen. Akten des 16. Linguistischen Kolloquiums, Kiel 1991, Tübingen, Niemeyer, pp. 187-196.

WAUGH, C. (1947): The Comics, New York, Macmillan.

WiLss, W. (1989): Anspielungen. Zur Manifestation von Kreativität und Routine in der Sprachverwendung, Tübingen, Niemeyer.

WÜrstle, R. (1991): “Äquivalenzprobleme bei der Übersetzung multimedialer Texte. Zur Übersetzung der Comics Les Frustrés von Claire Brétecher”, in Sснмітт, C. (Hg) Neue Methoden der Sprachmittlung, Wilhelmsfeld, Egert, pp. 149-170.

Zanettin, F. (1998): "Fumetti e traduzione multimediale", inTRAlinea 1, <http://www.intralinea.it/intra/voll/zanettin.htm>. 